\title{
A NEW CFA INTERPOLATION TECHNIQUE FOR SINGLE-SENSOR DIGITAL CAMERAS
}

\author{
R. Lukac and K.N. Plataniotis \\ Bell Canada Multimedia Laboratory, The Edward S. Rogers Sr. Department of ECE, \\ University of Toronto, 10 King's College Road, Toronto, Canada \\ lukacr@ieee.org,kostas@dsp.utoronto.ca
}

\begin{abstract}
A new color filter array (CFA) interpolation technique for single-sensor digital cameras is introduced. The proposed method utilizes an edge-sensing interpolation mechanism in conjunction with color-difference model based correction steps performed for each color channel. Thus, the proposed computationally efficient interpolation scheme is able to overcome the limitations of existing CFA based image acquisition solutions and restore color images without introducing false colors, edge blurring or visual artifacts. This produces excellent results in terms of subjective and objective image quality measures, while outperforming widely used CFA interpolation methods.
\end{abstract}

\section{INTRODUCTION}

The advances in color image processing [12] have allowed capturing and reproducing of real scenes in color as never before. Digital cameras for still images are among the most popular acquisition devices, whose commercial proliferation has a significant impact on the research in this area.

Digital color cameras acquire color information [8] by transmitting the image through Red (R), Green (G) and Blue (B) color filters having different spectral transmittances and then sampling the resulted images using three electronic sensors, usually charge coupled devices (CCD) and complementary metal oxide semiconductor (CMOS) sensors. To reduce cost and complexity, digital camera manufacturers use a single $\mathrm{CCD} / \mathrm{CMOS}$ sensor with a color filter array (CFA) to capture all the three primary colors $(\mathrm{R}, \mathrm{G}, \mathrm{B})$ at the same time. The Bayer pattern (Fig.1) [2], a widely used CFA, provides the array or mosaic of the RGB colors so that only one color element is available in each pixel. Two missing colors must be estimated from the adjacent pixels; this process refers to CFA interpolation, or demosaicing [4],[9].

\section{PROBLEM FORMULATION}

Let us consider, a $K_{1} \times K_{2}$ gray-scale image $z: Z^{2} \rightarrow Z$ representing a two-dimensional matrix of integer samples.



Fig. 1. Bayer color filter array.

In the Bayer CFA pattern, half of the pixels $z_{i} \in Z^{2}$, for $i=$ $1,2, \ldots, K_{1} K_{2}$, correspond to the $\mathrm{G}$ channel, whereas R,B channels are assigned the other half of the pixels. Assuming that $p=1,2, \ldots, K_{1}$ and $q=1,2, \ldots, K_{2}$ denote the spatial position of the pixels in vertical (image rows) and horizontal (image columns) directions, gray-scale pixels $z_{i}$ can be transformed into the RGB vectors $\mathbf{x}_{i}=\left(x_{i 1}, x_{i 2}, x_{i 3}\right) \in$ $Z^{2}$, for $i=(p-1) K_{2}+q$, as follows [10]:

$$
\mathbf{x}_{i}= \begin{cases}\left(z_{i}, 0,0\right) & \text { for } p \text { odd and } q \text { even } \\ \left(0,0, z_{i}\right) & \text { for } p \text { even and } q \text { odd } \\ \left(0, z_{i}, 0\right) & \text { otherwise }\end{cases}
$$

This transformation forms a $K_{1} \times K_{2}$ RGB image $\mathbf{x}: Z^{2} \rightarrow$ $Z^{3}$ representing a two-dimensional matrix of three-component samples [8]. Note that the color vectors $\mathbf{x}_{i}$ relate to one true component varying in $k$ from position to position, whereas other two components of $\mathbf{x}_{i}$ are set to zero. Therefore, it is desirable to estimate the missing color components of $\mathbf{x}$ and constitute the interpolated RGB image $\mathbf{y}: Z^{2} \rightarrow$ $Z^{3}$ to be as close to the desired RGB image $\mathbf{o}: Z^{2} \rightarrow Z^{3}$ as possible. This problem can be expressed through the minimization of the expected value of mean square error as follows [13]:

$$
\text { minimize } E\left\{\|\mathbf{o}-\mathbf{y}\|^{2}\right\}
$$

where $E\{\cdot\}$ indicates statistical expectation guaranteing the minimum average loss or risk.

To estimate the missing color components of $\mathbf{x}$, a sliding supporting window $W=\left\{\mathbf{x}_{i} \in Z^{2} ; i=0,1, \ldots, N-1\right\}$ of finite size $N$ is considered with the sample under consideration, sample $\mathbf{x}_{0}$, placed in the center of the window (Fig.2a). The procedure replaces the center $\mathbf{x}_{0}$ by some function of the local neighborhood area $\left\{\mathbf{x}_{1}, \mathbf{x}_{2}, \ldots, \mathbf{x}_{N-1}\right\}$ 


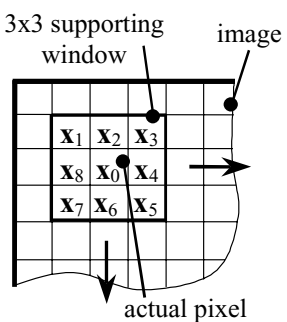

(a)

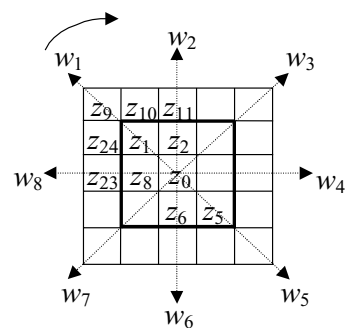

(b)
Fig. 2. Utilized components: (a) sliding supporting window, (b) edge-sensing weighting coefficients.

at a time. The rationale of this approach is to minimize the local distortion and ensure the stationarity of the processes generating the image [8].

\section{PROPOSED METHOD}

To follow structural information and interpolate missing image components in sharp shapes, efficient interpolation algorithms incorporate edge information into the interpolation process. The proposed method utilizes the edge-sensing mechanism of the C2D2 scheme [7]:

$$
\begin{gathered}
w_{1}=\frac{1}{1+\left(\left|z_{0}-z_{9}\right|+\left|z_{1}-z_{5}\right|\right) /(2 \sqrt{2})} \\
w_{2}=\frac{1}{1+\left(\left|z_{0}-z_{11}\right|+\left|z_{2}-z_{6}\right|\right) / 2}
\end{gathered}
$$

where $w_{1}$ and $w_{2}$ denote weights in north-west and north directions and $z_{0}, z_{1}, \ldots, z_{24}$ are original gray-scale values of $z$ (Fig.2b). Coefficients $w_{3}, w_{5}, w_{7}$ and $w_{4}, w_{6}, w_{8}$ are calculated applying appropriately the concept of (3) and (4), respectively.

Using 8 weighting coefficients, the $\mathrm{G}$ channel is interpolated as follows:

$$
\bar{y}_{02}= \begin{cases}x_{02} & \text { if } z_{0} \cong x_{02} \\ \sum_{i=1}^{N-1} w_{i}^{\prime} x_{i 2}^{\prime} & \text { otherwise }\end{cases}
$$

where $N=9$ relates to a $3 \times 3$ sliding window, $z_{0}$ is the acquired pixel before the transformation (1) in the same spatial position as the color (RGB) vector $\mathbf{x}_{i}$, operator $\cong \mathrm{de}$ notes a one to one relationship and $w_{i}^{\prime}=w_{i} / \sum_{j=1}^{N-1} w_{j}$ is the normalized weighting coefficient corresponding to predetermined $\mathrm{G}$ values $x_{i 2}^{\prime}$. For illustration purposes, quantities $x_{(1) 2}^{\prime}$ and $x_{(2) 2}^{\prime}$ are given by:

$$
\begin{aligned}
& x_{(1) 2}^{\prime}=\left(x_{(2) 2}+x_{(8) 2}\right) / 2+ \\
& \left(\left(z_{1}-z_{5}\right) /(2 \sqrt{2})+\left(z_{11}-z_{0}+z_{23}-z_{0}\right) / 4\right) / 2
\end{aligned}
$$

$$
x_{(2) 2}^{\prime}=x_{(2) 2}+\left(z_{11}-z_{0}+z_{2}-z_{6}\right) / 4
$$

Using the difference plane model [1] and the $G$ values obtained in (5), the R,B channels are estimated as follows:

$$
\bar{y}_{0 k}= \begin{cases}x_{0 k} & \text { if } z_{0} \cong x_{0 k} \\ \bar{y}_{02}+f_{(2 i) k}^{\prime \prime} & \text { if } z_{0} \cong x_{02} \\ \bar{y}_{02}+f_{(2 i-1) k}^{\prime \prime \prime} & \text { if } z_{0} \cong x_{0(k \pm 2)}\end{cases}
$$

where $k=1$ and $k=3$ characterize the $\mathrm{R}$ and $\mathrm{B}$ channels, respectively; $f_{(2 i) k}^{\prime \prime}=\sum_{i=1}^{(N-1) / 2} w_{i}^{\prime \prime}\left(x_{(2 i) k}-\bar{y}_{(2 i) 2}\right)$ and $f_{(2 i-1) k}^{\prime \prime \prime}=\sum_{i=1}^{(N-1) / 2} w_{i}^{\prime \prime \prime}\left(x_{(2 i-1) k}-\bar{y}_{(2 i-1) 2}\right)$ are defined using normalized weights $w_{i}^{\prime \prime}=w_{2 i} / \sum_{j=1}^{(N-1) / 2} w_{2 j}$ corresponding to edges in north, east, south and west directions; and coefficients $w_{i}^{\prime \prime \prime}=w_{(2 i-1)} / \sum_{j=1}^{(N-1) / 2} w_{(2 j-1)}$ relate to diagonally positioned edges.

It has been observed [9] that introducing a correction mechanism into the interpolation process improves contrast and accuracy of the initially interpolated $G$ channel. Since the difference plane model of (8) is simple and efficient, it is also perfectly suited to a correction stage. Thus, $G$ values are corrected as follows [10]:

$$
y_{02}= \begin{cases}\bar{y}_{0 k}+g_{(2 i) k}^{\prime \prime} & \text { if } z_{0} \cong x_{0 k} \\ \bar{y}_{02} & \text { otherwise }\end{cases}
$$

where $g_{(2 i) k}^{\prime \prime}=\sum_{i=1}^{(N-1) / 2} w_{i}^{\prime \prime}\left(\bar{y}_{(2 i) 2}-\bar{y}_{(2 i) k}\right)$ is defined using the weighting coefficients $w_{i}^{\prime \prime}$ of (8).

Considering the corrected $G$ values of (9) the R,B update is completed using the proposed approach as follows:

$$
y_{0 k}= \begin{cases}y_{0 k} & \text { if } z_{0} \cong x_{0 k} \\ y_{02}+h_{(2 i) k}^{\prime \prime} & \text { if } z_{0} \cong x_{02} \\ y_{02}+h_{(2 i-1) k}^{\prime \prime \prime} & \text { if } z_{0} \cong x_{0(k \pm 2)}\end{cases}
$$

where $h_{(2 i) k}^{\prime \prime}=\sum_{i=1}^{(N-1) / 2} w_{i}^{\prime \prime}\left(\bar{y}_{(2 i) k}-y_{(2 i) 2}\right), h_{(2 i-1) k}^{\prime \prime \prime}=$ $\sum_{i=1}^{(N-1) / 2} w_{i}^{\prime \prime \prime}\left(\bar{y}_{(2 i-1) k}-y_{(2 i-1) 2}\right)$ are defined using the weighting coefficients of (8), [10].

\section{EXPERIMENTAL RESULTS}

To examine the performance of the proposed framework and facilitate comparisons with the state of-the-art CFA interpolation schemes, some widely used natural test color images shown in Fig.3 are utilized. All test images have been normalized to a standard size of $512 \times 512$ pixels with a 8 -bit per channel RGB representation.

The proposed method is compared with other CFA interpolation methods listed in Table 1. To measure similarity between the original, full RGB, image o and interpolated image $\mathbf{y}$, a number of different objective measures, based on the difference in the statistical distributions of the pixel values, can be utilized [12]. In this paper, the mean square 

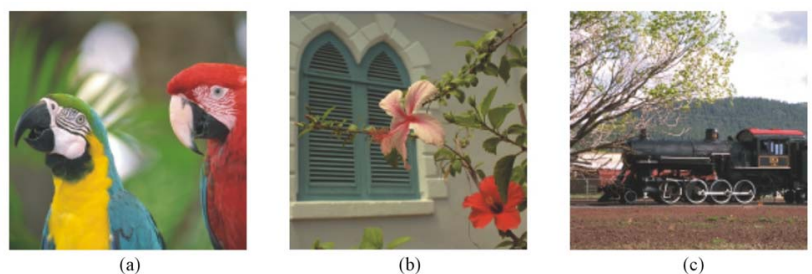

Fig. 3. Test color images: (a) Parrots, (b) Window, (c) Train.

Table 1. Methods taken for comparison with the proposed method.

\begin{tabular}{lll}
\hline Abbr. & Method & Ref. \\
\hline AP & Alternating projection approach & {$[4]$} \\
API & Adaptive color plane interpolation & {$[5]$} \\
BI & Bilinear interpolation & {$[11]$} \\
BD & Bilinear difference interpolation & {$[11]$} \\
C2D2 & Color correlation-directional derivatives & {$[7]$} \\
EMI & Edge map interpolation & {$[6]$} \\
MFI & Median filter interpolation & {$[3]$} \\
\hline
\end{tabular}

error (MSE) and the normalized color difference (NCD) are used to measure objectively the quality of the restored RGB image.

Tables 2-4 summarize results corresponding to the restoration of test images shown in Fig.3. Using natural test images we are able to compare performance of the methods in realistic applications, since in these images the correlation between the color channels vary significantly. It can be seen that the flexible design characteristics of the proposed method result in excellent performance as the reported error values indicate.

Figs.4-6 present zoomed parts of the restored images. These images allow for the comparison of the results corresponding to the fundamental BI and currently used API technique with those obtained through the proposed method in terms of a subjective (user-centered) evaluation. It is evident that the BI scheme fails near edges and produces color artifacts. The API scheme produces more quality results. However, the proposed method is capable of restoring the color images with the highest visual quality among the tested schemes and avoids color shifts and visual artifacts. This results in impressive visual quality of the restored images.

\section{CONCLUSIONS}

A new CFA interpolation approach for single-sensor digital cameras was provided. The proposed edge-sensing method utilizes interpolation and correction steps based on a refined
Table 2. Comparison of the presented algorithms using the test image Parrots.

\begin{tabular}{crrrc}
\hline Method & $M S E_{R}$ & $M S E_{G}$ & $M S E_{B}$ & $N C D$ \\
\hline AP & 4.588 & 3.806 & 8.151 & 0.0167 \\
API & 5.403 & 4.285 & 7.895 & 0.0173 \\
BI & 34.858 & 14.935 & 38.251 & 0.0262 \\
BD & 4.876 & 4.059 & 8.415 & 0.0170 \\
C2D2 & 5.446 & 5.242 & 7.099 & 0.0162 \\
EMI & 20.344 & 17.696 & 10.553 & 0.0207 \\
MFI & 7.090 & 2.909 & 10.433 & 0.0172 \\
Proposed & 3.909 & 2.490 & 6.261 & 0.0152 \\
\hline
\end{tabular}

Table 3. Comparison of the presented algorithms using the test image Window.

\begin{tabular}{crrrc}
\hline Method & $M S E_{R}$ & $M S E_{G}$ & $M S E_{B}$ & $N C D$ \\
\hline AP & 6.16 & 3.38 & 6.61 & 0.0212 \\
API & 7.03 & 4.53 & 7.11 & 0.0209 \\
BI & 41.48 & 17.24 & 41.61 & 0.0417 \\
BD & 7.06 & 4.57 & 7.92 & 0.0228 \\
C2D2 & 6.19 & 5.67 & 6.70 & 0.0193 \\
EMI & 12.42 & 9.80 & 11.33 & 0.0252 \\
MFI & 9.50 & 2.88 & 9.78 & 0.0239 \\
Proposed & 4.88 & 2.27 & 5.52 & 0.0181 \\
\hline
\end{tabular}

color difference model. Excellent design characteristics of the proposed method result in interpolated images which are sharp, naturally colored and pleasurable for viewing. Moreover, the new method outperforms previously developed interpolation methods in terms of commonly used objective criteria as well as the subjective evaluation.

\section{REFERENCES}

[1] J. Adams, "Design of practical color filter array interpolation algorithms for digital cameras," Proc. of the SPIE, vol. 3028, pp. 117-125, February 1997.

[2] B.E. Bayer, "Color imaging array," U.S. Patent 3971 065, 1976.

[3] W.T. Freeman, "Median filter for reconstructing missing color samples," U.S. Patent 5373 322, 1988.

[4] B. Gunturk, Y. Altunbasak, and R. Mersereau, "Color plane interpolation using alternating projections," IEEE Trans. Image Processing, vol. 11, pp. 997-1013, September 2002. 
Table 4. Comparison of the presented algorithms using the test image Train.

\begin{tabular}{crrrc}
\hline Method & $M S E_{R}$ & $M S E_{G}$ & $M S E_{B}$ & $N C D$ \\
\hline AP & 44.43 & 30.80 & 78.39 & 0.0560 \\
API & 109.24 & 96.62 & 145.01 & 0.0776 \\
BI & 517.71 & 254.59 & 607.19 & 0.1450 \\
BD & 74.71 & 65.45 & 113.47 & 0.0744 \\
C2D2 & 93.30 & 113.51 & 128.97 & 0.0702 \\
EMI & 159.42 & 152.53 & 195.69 & 0.0845 \\
MFI & 115.44 & 46.03 & 163.34 & 0.0798 \\
Proposed & 40.44 & 31.24 & 71.69 & 0.0503 \\
\hline
\end{tabular}

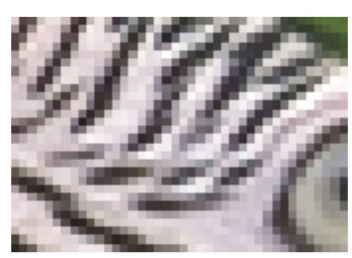

(a)

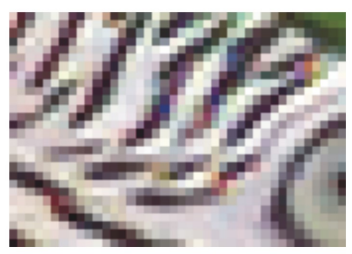

(c)

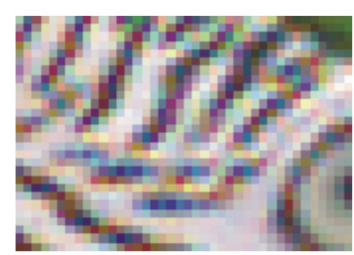

(b)

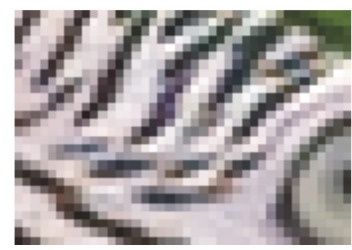

(d)
Fig. 4. Zoomed parts of the test image Parrots: (a) original image, (b) BI, (c) API, (d) proposed method.

[5] J.F. Hamilton and J.E. Adams, "Adaptive color plane interpolation in single sensor color electronic camera," U.S. Patent 5 629 734, 1997.

[6] B.S. Hur and M.G. Kang, "High definition color interpolation scheme for progressive scan CCD image sensor," IEEE Trans. Consumer Electronics, vol. 47, pp. 179-186, February 2001.

[7] N. Kehtarnavaz, H.J Oh, and Y. Yoo, "Color filter array interpolation using color correlations and directional derivatives," Journal of Electronic Imaging, vol. 12, pp. 621-632, October 2003.

[8] R. Lukac, B. Smolka, K. Martin, K.N. Plataniotis, and A.N. Venetsanopulos, "Vector filtering for color imaging," IEEE Signal Processing Magazine, SI Color Image Processing, to appear, April 2004.

[9] R. Lukac and K.N. Plataniotis, "A new color restoration solution for Bayer pattern based imaging devices," IEEE Signal Processing Letters, to appear, 2004.

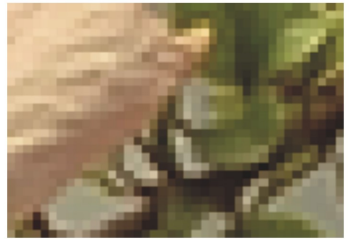

(a)

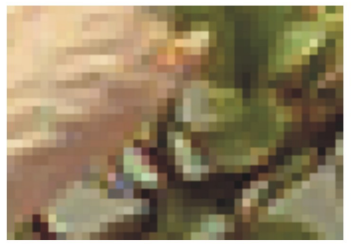

(c)

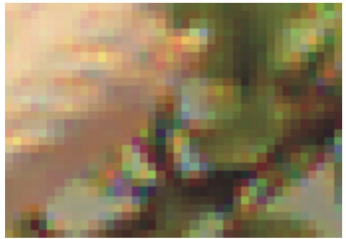

(b)

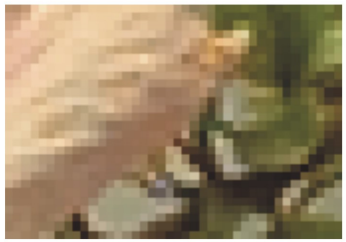

(d)
Fig. 5. Zoomed parts of the test image Window: (a) original image, (b) BI, (c) API, (d) proposed method.

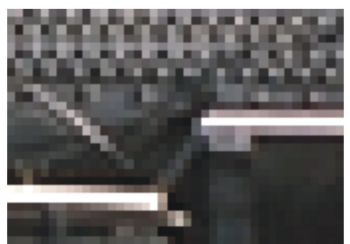

(a)

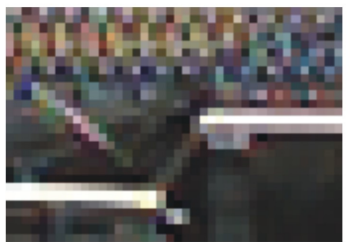

(c)

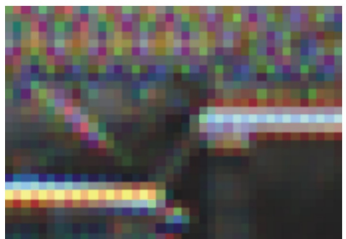

(b)

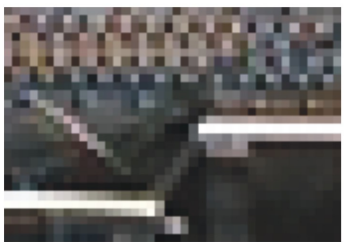

(d)
Fig. 6. Zoomed parts of the test image Train: (a) original image, (b) BI, (c) API, (d) proposed method.

[10] R. Lukac, K.N. Plataniotis, D. Hatzinakos, and M. Aleksic, "A new CFA interpolation framework," Signal Processing, submitted for publication.

[11] S.C. Pei and I.K. Tam, "Effective color interpolation in CCD color filter arrays using signal correlation," IEEE Trans. Circuits and Systems for Video Technology, vol. 13, pp. 503-513, June 2003.

[12] K.N. Plataniotis and A.N. Venetsanopoulos, Color Image Processing and Applications. Springer Verlag, 2000.

[13] H.J. Trussell and R.E. Hartwig, "Mathematics for demosaicking," IEEE Trans. Image Processing, vol. 11, pp. 485-492, April 2002. 